Pacific Journal of Mathematics

KOROVKIN SETS FOR AN OPERATOR ON A SPACE O 


\title{
KOROVKIN SETS FOR AN OPERATOR ON A SPACE OF CONTINUOUS FUNCTIONS
}

\author{
Le Baron O. Ferguson and Michael D. Rusk
}

We characterize Korovkin sets for sequences of either positive operators or contractive operators converging to an operator T. Properties of both the Korovkin sets and the operator $T$ are given which were previously known only in the case $T$ was the identity operator.

Let $C=C(Q)$ be the Banach space of continuous functions on a first countable, compact Hausdorff space $Q$. Let $\mathcal{J}$ be a subset of the bounded linear operators $\mathscr{B}(C)$ on $C$. A subspace $X$ of $C$ is said to be a $\mathscr{J}$-Korovkin set for an operator $T$ in $\mathscr{J}$ if for any sequence of operators $\left\{T_{n}\right\}$ in $\mathscr{J}$ the convergence of $T_{n} f$ to $T f$ in the uniform norm for all $f$ in $X$ implies the convergence of $T_{n} f$ to $T f$ for all $f$ in $C$. This paper is concerned with $\mathscr{J}$-Korovkin sets when $\mathscr{J}$ consists of either the positive $(f \geqq 0$ implies $T f \geqq 0)$ operators or the contractive $(\|T\| \leqq 1)$ operators in $\mathscr{B}(C)$.

The case where $T$ is the identity operator is now classical. See, for instance, Lorentz [3]. In this same paper the extension of the classical theory to the case of arbitrary operators $T$ is mentioned as an open problem. This extension is the subject of the present paper. In case $T$ is the identity operator our results reduce to the classical ones. A number of authors have considered the case where $T$ is a lattice homomorphism between (possibly distinct) vector lattices. The present situation is different since we consider operators with the same domain and range and assume the weaker condition that $T$ either be positive or have norm one. In addition, Cavaretta [1] and Micchelli $[4,5]$ have considered the case where $T$ is a positive operator, but not necessarily a lattice homomorphism.

Many of the following results have obvious analogues in the case where the operators are assumed to be both positive and contractive.

1. General theory. Korovkin-type theorems are usually stated for either sequences of positive operators or sequences of contractive operators. Some results about Korovkin sets can be shown in a more general setting. This observation has also been made by Micchelli [5].

For a bounded linear operator $T$, let $T^{*}$ denote the adjoint of $T$. For a point $q$ in $Q$, let $\hat{q}$ denote the functional in $C^{*}$ given by evaluation at the point $q$. Let $\mathscr{L}$ be a subset of $C^{*}$ and $\mathscr{J}$ be 
the set of all bounded linear operators $T$ on $C$ such that $T^{*} \hat{q}$ is in $\mathscr{L}$ for all $q$ in $Q$. For example, if $\mathscr{L}$ were the positive functionals on $C$, then $\mathscr{J}$ would be the positive operators.

Let $\lambda$ be a functional in $\mathscr{L}$ and $X$ be a subspace of $C$. We say $X$ is an $\mathscr{L}$-Korovkin set for $\lambda$ if for any sequence $\left\{\lambda_{n}\right\}$ in $\mathscr{L}$ the fact that $\lambda_{n}(f) \rightarrow \lambda(f)$ for all $f$ in $X$ implies $\lambda_{n}(f) \rightarrow \lambda(f)$ for all $f$ in $C$. We say $X$ is an $\mathscr{L}$-determining set for $\lambda$ if for any $\mu$ in $\mathscr{L}$ the equality $\mu(f)=\lambda(f)$ for all $f$ in $X$ implies $\mu=\lambda$. The latter concept was first introduced for operators by Šaškin [8]. These two concepts are equivalent in the following sense.

THEOREM 1.1. Let $\mathscr{L}$ be a weak* closed subset of $C^{*}$ and let $\lambda$ be a functional in $\mathscr{L}$. A subset $X$ of $C$ has the property that for any norm bounded sequence $\left\{\lambda_{n}\right\}$ in $\mathscr{L}$ the fact that $\lambda_{n}(f) \rightarrow \lambda(f)$ for all $f$ in $X$ implies $\lambda_{n}(f) \rightarrow \lambda(f)$ for all $f$ in $C(X$ is a Korovkin set for norm bounded sequences in $\mathscr{L}$ converging to $\lambda$ ) if and only if $X$ is an $\mathscr{L}$-determining set for $\lambda$.

Proof. Suppose $X$ is a Korovkin set for norm bounded sequences in $\mathscr{L}$ converging to $\lambda$. Let $\mu$ be any functional in $\mathscr{L}$ such that $\mu(f)=\lambda(f)$ for all $f$ in $X$. Define $\lambda_{n}=\mu$ for $n=1,2, \cdots$. Then $\left\{\lambda_{n}\right\}$ is a norm bounded sequence in $\mathscr{L}$ such that $\lambda_{n}(f) \rightarrow \lambda(f)$ for all $f$ in $X$. By assumption $\lambda_{n}(f) \rightarrow \lambda(f)$ for all $f$ in $C$. Therefore $\mu(f)=\lambda(f)$ for all $f$ in $C$. Hence, $X$ is an $\mathscr{L}$-determining set for $\lambda$.

On the other hand, suppose $X$ is an $\mathscr{L}$-determining set for $\lambda$. Let $\left\{\lambda_{n}\right\}$ be a norm bounded sequence in $\mathscr{L}$ such that $\lambda_{n}(f) \rightarrow \lambda(f)$ for all $f$ in $X$. By the Banach-Alaoglu theorem there exists a weak* limit point $\mu$ of $\left\{\lambda_{n}\right\}$. Since $\mu(f)=\lambda(f)$ for all $f$ in $X$ and $X$ is an $\mathscr{L}$-determining set for $\lambda$, we have $\mu=\lambda$. The sequence $\left\{\lambda_{n}\right\}$ is contained in a compact set and has the unique limit point $\lambda$, hence the sequence converges to $\lambda$. Thus $\lambda_{n}(f) \rightarrow \lambda(f)$ for all $f$ in $C$ and the theorem is proved.

Another fundamental result is the relation between $\mathscr{J}$-and $\mathscr{L}$ Korovkin sets.

Theorem 1.2. For a convex subset $\mathscr{L}$ of $C^{*}$ we define $\mathscr{J}$ as above. Let $X$ be a subspace of $C$ and $T$ an operator in $\mathscr{J}$. Then $X$ is a $\mathscr{J}$-Korovkin set for $T$ if and only if $X$ is an $\mathscr{L}$-Korovkin set for $T^{*} \hat{q}$ where $q$ is any point in $Q$.

Proof. Suppose $X$ is a $\mathscr{J}$-Korovkin set for $T$. Let $q$ be a point in $Q$. Suppose there is a sequence $\left\{\lambda_{n}\right\}$ in $\mathscr{L}$ such that $\lambda_{n}(f) \rightarrow$ $\left(T^{*} \hat{q}\right)(f)$ for $f$ in $X$. We show $\lambda_{n}(f) \rightarrow\left(T^{*} \hat{q}\right)(f)$ for all $f$ in $C$ by constructing a sequence of operators in $\mathscr{J}$. Let the sequence $\left\{U_{n}\right\}$ 
be a decreasing neighborhood base at the point $q$. This is possible since $Q$ is first countable. By Urysohn's lemma there exists, for each $n \geqq 1$, a continuous function $h_{n}: Q \rightarrow[0,1]$ such that $h_{n}(q)=1$ and $h_{n}(p)=0$ for $p$ not in $U_{n}$. Define the operator $T_{n}$ in $\mathscr{B}(C)$ by

$$
\left(T_{n} f\right)(p)=h_{n}(p) \lambda_{n}(f)+\left(1-h_{n}(p)\right)(T f)(p) \text {. }
$$

It follows from the definition of $\mathscr{J}$ and the convexity of $\mathscr{L}$ that $T_{n}$ is in $\mathcal{J}$ for all $n \geqq 1$. Fix $\varepsilon>0$ and $f$ in $X$. Since $T f$ is a continuous function, there exists a set, say $U_{m}$, in the neighborhood base of the point $q$ such that

$$
|(T f)(q)-(T f)(p)|<\varepsilon / 2
$$

for all $p$ in $U_{m}$. Also $h_{n}(p)=0$ for any $p$ not in $U_{m}$ and any $n \geqq$ $m$. Hence, for any $p$ in $Q$ and any $n \geqq m$ we have

$$
h_{n}(p)|(T f)(p)-(T f)(q)|<\varepsilon / 2 .
$$

By assumption there exists $j \geqq 1$ such that for all $n \geqq j$

$$
\left|\lambda_{n}(f)-(T f)(q)\right|<\varepsilon / 2 \text {. }
$$

Using the definition of $T_{n}$ and letting $k$ be the maximum of $j$ and $m$, we have for all $n \geqq k$

$$
\begin{aligned}
\left|\left(T_{n} f\right)(p)-(T f)(p)\right| & =\left|h_{n}(p) \lambda_{n}(f)-h_{n}(p)(T f)(p)\right| \\
& =h_{n}(p)\left|\lambda_{n}(f)-(T f)(p)\right| \\
& \leqq h_{n}(p)\left(\left|\lambda_{n}(f)-(T f)(q)\right|+|(T f)(q)-(T f)(p)|\right) \\
& <\varepsilon / 2+\varepsilon / 2=\varepsilon .
\end{aligned}
$$

Since $X$ is a $\mathscr{J}$-Korovkin set for $T, T_{n} f \rightarrow T f$ for all $f$ in $C$. In particular,

$$
\lambda_{n}(f)=\left(T_{n} f\right)(q) \rightarrow(T f)(q)=\left(T^{*} \hat{q}\right)(f) .
$$

This shows that $X$ is an $\mathscr{L}$-Korovkin set for $T^{*} \hat{q}$ for all $q$ in $Q$.

On the other hand, suppose $X$ is an $\mathscr{L}$-Korovkin set for $T^{*} \hat{q}$ for all $q$ in $Q$. Let $\left\{T_{n}\right\}$ be a sequence of operators in $\mathscr{J}$ such that $T_{n} f \rightarrow T f$ for all $f$ in $X$. As in Šaškin [7] we use the wellknown result that a sequence $\left\{f_{n}\right\}$ in $C$ converges uniformly to $f$ in $C$ if and only if for any sequence $\left\{q_{n}\right\}$ converging to a point $q$ in $Q$ it follows that $f_{n}\left(q_{n}\right) \rightarrow f(q)$. Let $\left\{q_{n}\right\}$ be a sequence in $Q$ converging to a point $q$ in $Q$. For all $f$ in $X,\left(T_{n} f\right)\left(q_{n}\right) \rightarrow(T f)(q)$ or $\left(T_{n}^{*} \hat{q}_{n}\right)(f) \rightarrow$ $\left(T^{*} \hat{q}\right)(f)$. By assumption this implies $\left(T_{n}^{*} \widehat{q}_{n}\right)(f) \rightarrow\left(T^{*} \hat{q}\right)(f)$ for all $f$ in $C$. By the same result we now have $T_{n} f \rightarrow T f$ for all $f$ in $C$. Hence, $X$ is a $\mathscr{J}$-Korovkin set for $T$ and the theorem is proved. 
2. Positive operators. In this section we give two characterizations of Korovkin sets for positive operators.

Let $\mathscr{J}_{+}$denote the positive bounded linear operators on $C$ and let $\mathscr{L}_{+}$denote the positive bounded linear functionals on $C$. For a subspace $X$ of $C$ and a functional $\mu$ in $C^{*}$, let $\left.\mu\right|_{X}$ be the functional in $X^{*}$ obtained by restricting $\mu$ to $X$. We define $M=\left\{\left.\hat{p}\right|_{X}: p \in Q\right\}$.

In Micchelli [5] and Grossman [2], the authors assume that a $\mathscr{J}_{+}$-Korovkin set contains a strictly positive function. The following theorem (compare to Šaškin [7, Lemma 1]) shows this hypothesis to be unnecessary.

TheOREm 2.1. If a subspace $X$ of $C$ is an $\mathscr{L}_{+}$-Korovkin set for a functional $\mu$ in $\mathscr{L}_{+}$, then $X$ contains a strictly positive function.

Proof. Suppose $X$ is an $\mathscr{L}_{+}$-Korovkin set for a functional $\mu$ in $\mathscr{L}_{+}$. Let $\overline{\operatorname{co}}(M)$ be the weak* closure of the convex hull of $M$. We claim $\overline{\operatorname{co}}(M)$ does not contain $\left.0\right|_{X}$, the zero functional restricted to $X$. We assume the claim is false and arrive at a contradiction. Let $\left\{\mu_{\alpha}\right\}$ be a net in $\operatorname{co}(M)$, the convex hull of $M$, such that $\left.\mu_{\alpha} \rightarrow 0\right|_{X}$ in the weak* topology of $X^{*}$. For each $\alpha$ there exists a positive integer $n \beta_{i} \geqq 0$ and $q_{i}$ in $Q, 1 \leqq i \leqq n$, where $\sum_{i=1}^{n} \beta_{i}=1$, such that

$$
\mu_{\alpha}=\left.\sum_{i=1}^{n} \beta_{i} \widehat{q}_{i}\right|_{X}
$$

We define the natural extension $\nu_{\alpha}=\sum_{i=1}^{n} \beta_{i} \hat{q}_{i}$ in $C^{*}$. Note that $\nu_{\alpha}$ is a positive functional where $\nu_{\alpha}(1)=1$ and therefore $\left\|\nu_{\alpha}\right\|=1$. By the Banach-Alaoglu theorem there exists a weak* limit point $\nu$ of $\left\{\nu_{\alpha}\right\}$. Clearly, $\nu$ is a positive functional in $C^{*}$ with $\left.\nu\right|_{X}=\left.0\right|_{X}$, but $\nu \neq 0$ since $\nu(1)=1$. Then for the sequence $\left\{\lambda_{n}\right\}$ in $\mathscr{L}^{+}$given by $\lambda_{n}=\nu+\mu$, we have $\lambda_{n}(f) \rightarrow \mu(f)$ for all $f$ in $X$. However, $\lambda_{n}(1)=$ $\nu(1)+\mu(1)=1+\mu(1)$ does not converge to $\mu(1)$. This contradicts the hypothesis of the theorem. So the claim is true. By a standard separation theorem there exists a weak closed hyperplane $H$ separating $\left.0\right|_{X}$ and $\overline{c o}(M)$, i.e., there exists $g$ in $X$ and $\beta>0$ such that $g(p)>\beta$ for all $p$ in $Q$. The theorem is proved.

THEOREM 2.2. A subspace $X$ of $C$ is a $\mathscr{J}_{+}$-Korovkin set for an operator $T$ in $\mathscr{J}_{+}$if and only if $X$ is an $\mathscr{L}_{+}$-determining set for $T^{*} \hat{q}$ for all $q$ in $Q$.

Proof. Suppose $X$ is a $\mathscr{J}_{+}$-Korovkin set for $T$. By Theorem $1.2, X$ is an $\mathscr{L}_{+}$-Korovkin set for $T^{*} \hat{q}$ for all $q$ in $Q$. Therefore, as in Theorem 1.1, $X$ is also an $\mathscr{L}_{+}$-determining set for $T^{*} \hat{q}$ for all 
$q$ in $Q$.

Conversely, suppose $X$ is an $\mathscr{L}_{+}$-determining set for $T^{*} \hat{q}$ for all $q$ in $Q$. By Theorem 2.1, there exists $g$ in $X$ such that $g(q) \geqq 1$ for all $q$ in $Q$. Fix a point $q$ in $Q$. We claim $X$ is an $\mathscr{L}_{+}$-Korovkin set for $T^{*} \hat{q}$. Let $\left\{\lambda_{n}\right\}$ be a sequence in $\mathscr{L}_{+}$such that $\lambda_{n}(f) \rightarrow\left(T^{*} \hat{q}\right)(f)$ for all $f$ in $X$. In particular, $\lambda_{n}(g) \rightarrow\left(T^{*} \hat{q}\right)(g)=(T g)(q)$. Since $\lambda_{n}$ is a positive functional for each $n \geqq 1$

$$
\left\|\lambda_{n}\right\|=\lambda_{n}(1) \leqq \lambda_{n}(g) \rightarrow(T g)(q) .
$$

Therefore, the sequence $\left\{\lambda_{n}\right\}$ is norm bounded. By Theorem 1.1, the claim is true. The result now follows from Theorem 1.2. The theorem is proved.

If the subspace $X$ is finite dimensional, then a more geometric condition is possible. When $T$ is the identity operator, Corollary 2.3 is essentially Lorentz's Theorem 4 [3].

Corollary 2.3. An m-dimensional subspace $X$ is a $\mathscr{J}_{+}$-Korovkin set for an operator $T$ in $\mathscr{J}_{+}$if and only if $X$ satisfies the condition that $\left.0\right|_{X}$ is not in $\operatorname{co}(M)$ and for any subset $\left\{q, q_{1}, q_{2}, \cdots, q_{m+1}\right\}$ in $Q$ and $\beta_{i} \geqq 0$ for $1 \leqq i \leqq m+1$ the equality $\left.\sum_{i=1}^{m+1} \beta_{i} \widehat{q}_{i}\right|_{X}=\left.\left(T^{*} \widehat{q}\right)\right|_{X}$ implies $\sum_{i=1}^{m+1} \beta_{i} \hat{q}_{i}=T^{*} \hat{q}$.

Proof. The necessity of the condition follows directly from Theorem 2.2.

We now show the condition is sufficient. Suppose $X$ satisfies the condition of the theorem, but $X$ is not a $\mathscr{J}_{+}$-Korovkin set for $T$. By Theorem 2.2 there exists a positive functional $\mu$ in $C^{*}$ such that for some $q$ in $Q$ we have $\mu(f)=\left(T^{*} \hat{q}\right)(f)$ for all $f$ in $X$, but for some $g$ in $C$ we have $\mu(g) \neq\left(T^{*} \hat{q}\right)(g)$. Let $Y$ be the subspace of $C$ spanned by $X$ and $g$. Let $M_{1}$ be the set of point evaluations in $Y^{*}$. The functional $\left.0\right|_{Y}$ is not in $\operatorname{co}\left(M_{1}\right)$, since $\left.0\right|_{X}$ is not in $\operatorname{co}(M)$. By a known theorem there exists $\alpha_{i} \geqq 0$ and $q_{i}$ in $Q$ for $1 \leqq i \leqq m+1$ such that

$$
\mu(f)=\sum_{\imath=1}^{m+1} \alpha_{\imath} \widehat{q}_{\imath}(f)
$$

for all $f$ in $Y$. Thus,

$$
\sum_{i=1}^{m+1} \alpha_{i} \widehat{q}_{i}(f)=\left(T^{*} \hat{q}\right)(f)
$$

for all $f$ in $X$, but not for $f=g$. This contradicts our assumption. Hence, $X$ must be a $\mathscr{J}_{+}$-Korovkin set for $T$. The corollary is proved. If a positive operator $T$ has an $m$-dimensional $\mathscr{J}_{+}$-Korovkin set, 
then Micchelli [5] has shown that $T$ must be finitely defined of order $m$. This means that for every $q$ in $Q$ there exists $\alpha_{i} \geqq 0$ and $q_{i}$ in $Q$ for $1 \leqq i \leqq m$ such that $T^{*} \hat{q}=\sum_{i=1}^{m} \alpha_{i} q_{i}$. We shall show a similar result for contractive operators in the next section.

3. Contractive operators. In this section we prove two characterizations of contractive operator Korovkin sets. We also establish two properties of any operator $T$ having a contractive operator Korovkin set. Finally, we give conditions under which positive operator Korovkin sets are equivalent to contractive operator Korovkin sets. These results are all stated for an operator $T$ of norm one. If $T$ has norm $c>0$, it is easy to verify the corresponding theorems for sequences of operators of norm at most $c$.

Let $\mathscr{J}^{1}$ denote the bounded linear operators on $C$ of norm at most one and let $\mathscr{L}^{1}$ denote the bounded linear functionals on $C$ of norm at most one. The following result can be compared with Šaškin [9].

THEOREM 3.1. Let $T$ be a norm one bounded linear operator. $A$ subspace $X$ of $C$ is a $\mathcal{J}^{1}$-Korovkin set for $T$ if and only if $X$ is an $\mathscr{L}^{1}$-determining set for $T^{*} \hat{q}$ for all $q$ in $Q$.

Proof. The proof follows directly from Theorems 1.1 and 1.2.

The following is the analogue of Saškin's Theorem 2 [9]. This condition seems to be necessarily more complicated than Šaškin's condition since for $q$ in $Q$ the functional $T^{*} \hat{q}$ is not necessarily a point evaluation.

COROLlaRY 3.2. Let $T$ be an operator in $\mathscr{B}(C)$ of norm one. An m-dimensional subspace $X$ of $C$ is a $\mathscr{J}^{1}$-Korovkin set for $T$ if and only if $X$ satisfies the condition that, for any subset $\left\{q, q_{1}, \cdots\right.$, $\left.q_{m+2}\right\}$ in $Q$ and for any functional $\mu=\sum_{i=1}^{m+2} \beta_{i} \widehat{q}_{i}$ where $\beta_{i}$ is in $\boldsymbol{R}$ and $\sum_{i=1}^{m+2}\left|\beta_{i}\right|=1$, the equality $\left.\mu\right|_{X}=\left.\left(T^{*} \hat{q}\right)\right|_{X}$ implies $\mu=T^{*} \hat{q}$.

The proof of this corollary is similar to the proof of Corollary 2.3 .

If an operator $T$ has a $\mathscr{J}^{1}$-Korovkin set $X$, then $T^{*} \hat{q}$ for each $q$ in $Q$ must have certain properties. First, we note that $T$ must be a finitely defined operator if $X$ is finite dimensional.

Corollary 3.3. If the m-dimensional subspace $X$ of $C$ is a $\mathscr{J}^{1}$-Korovkin set for the norm one operator $T$ in $\mathscr{B}(C)$, then $T$ is finitely defined of order $m+1$.

Proof. Suppose $T$ and $X$ satisfy the hypotheses of the theorem. 
For each $q$ in $Q$ there exist $q_{1}, q_{2}, \cdots, q_{m+1}$ in $Q$ and $\alpha_{i}$ in $\boldsymbol{R}$ where $\sum_{i=1}^{m+1}\left|\alpha_{i}\right|=1$ such that for any $f$ in $X$

$$
\left(T^{*} \hat{q}\right)(f)=\sum_{i=1}^{m+1} \alpha_{i} \hat{q}_{i}(f) .
$$

Since $X$ is a Korovkin set, by Theorem 3.1, the above equality holds for all $f$ in $C$. Therefore $T$ is finitely defined of order $m+1$. The corollary is proved.

In the following Corollary we have another condition on $\left.\left(T^{*} \hat{q}\right)\right|_{X}$.

CoRollary 3.4. Let $T$ be a norm one operator in $\mathscr{B}(X)$. Let $X$ be a closed proper subspace of $C$. If $X$ is a $\mathcal{J}^{1}$-Korovkin set for $T$, then for all $q$ in $Q$ we have

$$
\left\|\left.T^{*} \hat{q}\right|_{X}\right\|_{X^{*}}=\left\|T^{*} \hat{q}\right\|=1
$$

where

$$
\left\|\left.\left(T^{*} \hat{q}\right)\right|_{X}\right\|_{X^{*}}=\sup \left\{\left|\left(T^{*} \hat{q}\right)(f)\right|: f \in X,\|f\| \leqq 1\right\} .
$$

Proof. Let $\alpha=\left\|\left.T^{*} \hat{q}\right|_{X}\right\|_{X^{*}}$. Then $\alpha \leqq\left\|T^{*} \hat{q}\right\| \leqq 1$. Suppose $\alpha<$ 1. Then by the Hahn-Banach theorem $\left.\left(T^{*} \hat{q}\right)\right|_{X}$ has an extension to $C$ of norm $\alpha$. By a modification (see Rusk [6]) of the proof of the Hahn-Banach theorem one shows that $\left.\left(T^{*} \hat{q}\right)\right|_{X}$ has an extension to $C$ of norm $\beta$ where $\alpha<\beta \leqq 1$. This contradicts Theorem 3.1. Therefore $\alpha=1$, which proves the corollary.

A natural question arises about subspaces which are contractive Korovkin sets for any finitely defined operator of a given order. Cavaretta [1] has given such sets for the positive operators. The next corollary shows that there are no such finite dimensional subspaces for contractive operators.

CoROLlary 3.5. Let $Q$ also be nondiscrete. Then $C$ has no finite dimensional $\mathcal{J}^{1}$-Korovkin set for all finitely defined operators $T$ of order 2 such that $\left\|T^{*} \hat{q}\right\|=1$ for all $q$ in $Q$.

Proof. Suppose the theorem is false. Then there exists a finite dimensional subspace $X$ of $C$ which is such a set. For any distinct points $q_{1}$ and $q_{2}$ in $Q$ and $\beta_{1}$ and $\beta_{2}$ in $R$ such that $\left|\beta_{1}\right|+\left|\beta_{2}\right|=1$ there is some operator $T$ as above such that $T^{*} \hat{q}=\beta_{1} \hat{q}_{1}+\beta_{2} \hat{q}_{2}$. By Corollary 3.4, $\left\|\left.\left(T^{*} \hat{q}\right)\right|_{X}\right\|_{X^{*}}=1$. Since the closed unit ball of $X$ is compact, there exists $f$ in $X$ such that $\left(T^{*} \hat{q}\right)(f)=1=\beta_{1} f\left(q_{1}\right)+\beta_{2} f\left(q_{2}\right)$ and $\|f\| \leqq 1$. This can happen only if $f\left(q_{1}\right)=\operatorname{sgn} \beta_{1}$ and $f\left(q_{2}\right)=$ $\operatorname{sgn} \beta_{2}$, where $\operatorname{sgn} x=x /|x|$ if $x \neq 0$. Since $Q$ is not discrete, there exists a sequence $\left\{q_{n}\right\}$ in $Q$ such that $q_{n} \rightarrow q$ in $Q$, and such that $q_{n} \neq q$ for $n \geqq 1$. Then according to the previous argument there 
exist $f_{n}$ in $C$ of norm one such that $f_{n}\left(q_{n}\right)=1$, but $f_{n}(q)=-1$ for $n \geqq 1$. Again since the closed unit ball of $X$ is compact, there exists a subsequence $\left\{n_{j}\right\}$ such that $f_{n_{j}} \rightarrow f$ uniformly as $j \rightarrow \infty$ for some $f$ in $X$. From the uniform convergence we have $1=f_{n_{j}}\left(q_{n_{j}}\right) \rightarrow f(q)$ as $j \rightarrow \infty$. On the other hand $-1=f_{n_{j}}(q) \rightarrow f(q)$ as $j \rightarrow \infty$. This contradiction implies the corollary is true.

Suppose $X$ is a finite dimensional subspace of $C$ containing the constants. Lorentz [3, Theorem 8] has shown that $X$ is a $\mathscr{J}_{+}$Korovkin set for the identity operator if and only if $X$ is a $\mathscr{J}^{1}$ Korovkin set for the identity. We extend this result in the next theorem.

THEOREM 3.6. Let $T$ be a norm one positive operator in $\mathscr{B}(C)$ and let $X$ be a subspace of $C$ containing the constants. The subspace $X$ is a $\mathscr{J}_{+}$-Korovkin set for $T$ and $T 1=1$ if and only if $X$ is a $\mathcal{J}^{1}$-Korovkin set for $T$.

Proof. Suppose $X$ is a $\mathscr{J}_{+}$-Korovkin set for $T$ and $T 1=1$. Suppose for a point $q$ in $Q$ and a functional $\mu$ in $\mathscr{L}^{1}$ that $\left.\mu\right|_{X}=$ $\left.\left(T^{*} \hat{q}\right)\right|_{X}$. From

$$
\mu(1)=(T 1)(q)=1=\|\mu\|
$$

it follows that $\mu$ is a positive functional. By Theorem 2.2 we have $\mu=T^{*} \hat{q}$. Therefore, by Theorem 3.1, $X$ is a $\mathcal{J}^{1}$-Korovkin set for $T$.

Conversely, suppose $X$ is a $\mathscr{J}^{1}$-Korovkin set for $T$. By Corollary 3.4 and since $T$ is a positive operator $1=\left\|T^{*} \hat{p}\right\|=(T 1)(p)$ for all $p$ in $Q$, i.e., $T 1=1$. Suppose for a point $q$ in $Q$ and a functional $\mu$ in $\mathscr{L}_{+}$that $\left.\mu\right|_{X}=\left.\left(T^{*} \hat{q}\right)\right|_{X}$. Then

$$
\|\mu\|=\mu(1)=(T 1)(q)=1 .
$$

By Theorem 3.1 we have $\mu=T^{*} \hat{q}$. Therefore by Theorem 2.2, $X$ is a $\mathscr{J}_{+}$-Korovkin set for $T$. The theorem is proved.

The hypothesis that $T 1=1$ cannot be omitted in Theorem 3.6. Consider the positive norm one operator $T$ in $\mathscr{B}(C)$ where $Q=[0,1]$ defined by

$$
(T f)(q)=(1+q) f(q) / 3, \quad q \in[0,1] .
$$

If $X$ is spanned by $\left\{1, x, x^{2}\right\}$, then $X$ is a $\mathscr{J}_{+}$-Korovkin set for $T$ (see Cavaretta [1, Theorem 2]). However, since $\left\|T^{*} \hat{q}\right\|=1 / 3$ when $q=0$, by Corollary 3.4, $X$ is not a $\mathscr{J}^{1}$-Korovkin set for $T$. 


\section{REFERENCES}

1. A. S. Cavaretta, Jr., A Korovkin Theorem for Finitely Defined Operators, Approximation Theory, ed. G. G. Lorentz, Academic Press, New York, 1973, 299-305.

2. M. W. Grossman, Korovkin type theorems with respect to a Markov operator, Notices of the AMS, 22 (1975) A-463, Abstract 75T-B127.

3. G. G. Lorentz, Korovkin sets, Regional Conference at the Univ. of Calif., Riverside, June 15-19, 1972.

4. C. A. Micchelli, Chebyshev subspaces and convergence of positive linear operators, Proc. Amer. Math. Soc., 40 (1973), 448-452.

5 . Convergence of positive linear operators on $C(X)$, Private Communication, November, 1974.

6. M. D. Rusk, Korovkin type theorems for finitely defined operators, Disseration, Univ. of Calif., Riverside, March, 1975.

7. Yu. A. Šaškin, Korovkin systems in spaces of continuous functions, (Russian), Izv. Akad. Nauk SSSR, Ser, Mat., 26 (1962), 495-512, English transl., Amer. Math. Soc. Transl., (2) 54 (1966), 125-144.

8. - Finitely defined linear operators in spaces of continuous functions, (Russian) Uspeki Mat. Nauk, 20 (1965), no. 6 (126), 175-180.

9. - On the convergence of contraction operators, (Russian) Mathematica (Cluj), 11 (1969), 355-360.

10. D. Wulbert, Convergence of operators and Korovkin's theorem, J. Approximation Theory, 1 (1968), 381-390.

Received August 14, 1975 and in revised form April 6, 1976.

University of CALIFornia, Riverside

AND

UNIVERSity OF REDLANDS 



\section{PACIFIC JOURNAL OF MATHEMATICS}

\section{EDITORS}

RICHARD ARENS (Managing Editor)

University of California

Los Angeles, California 90024

\section{R. A. Beaumont}

University of Washington

Seattle, Washington 98105
J. DugundjI

Department of Mathematics

University of Southern California

Los Angeles, California 90007

D. Gilbarg and J. Milgram

Stanford University

Stanford, California 94305

\section{ASSOCIATE EDITORS}
E. F. BECKENBACH
B. H. NeumanN
F. WOLF
K. YosHIDA

\section{SUPPORTING INSTITUTIONS}

\author{
UNIVERSITY OF BRITISH COLUMBIA \\ CALIFORNIA INSTITUTE OF TECHNOLOGY \\ UNIVERSITY OF CALIFORNIA \\ MONTANA STATE UNIVERSITY \\ UNIVERSITY OF NEVADA \\ NEW MEXICO STATE UNIVERSITY \\ OREGON STATE UNIVERSITY \\ UNIVERSITY OF OREGON \\ OSAKA UNIVERSITY
}

\author{
UNIVERSITY OF SOUTHERN CALIFORNIA \\ STANFORD UNIVERSITY \\ UNIVERSITY OF HAWAII \\ UNIVERSITY OF TOKYO \\ UNIVERSITY OF UTAH \\ WASHINGTON STATE UNIVERSITY \\ UNIVERSITY OF WASHINGTON \\ AMERICAN MATHEMATICAL SOCIETY
}

The Supporting Institutions listed above contribute to the cost of publication of this Journal, but they are not owners or publishers and have no responsibility for its content or policies.

Mathematical papers intended for publication in the Pacific Journal of Mathematics should be in typed form or offset-reproduced, (not dittoed), double spaced with large margins. Please do not use built up fractions in the text of your manuscript. You may however, use them in the displayed equations. Underline Greek letters in red, German in green, and script in blue. The first paragraph or two must be capable of being used separately as a synopsis of the entire paper. Items of the bibliography should not be cited there unless absolutely necessary, in which case they must be identified by author and Journal, rather than by item number. Manuscripts, in triplicate, may be sent to any one of the editors. Please classify according to the scheme of Math. Reviews, Index to Vol. 39. All other communications should be addressed to the managing editor, or Elaine Barth, University of California, Los Angeles, California, 90024.

The Pacific Journal of Mathematics expects the author's institution to pay page charges, and reserves the right to delay publication for nonpayment of charges in case of financial emergency.

100 reprints are provided free for each article, only if page charges have been substantially paid. Additional copies may be obtained at cost in multiples of 50 .

The Pacific Journal of Mathematics is issued monthly as of January 1966. Regular subscription rate: $\$ 72.00$ a year (6 Vols., 12 issues). Special rate: $\$ 36.00$ a year to individual members of supporting institutions.

Subscriptions, orders for back numbers, and changes of address should be sent to Pacific Journal of Mathematics, 103 Highland Boulevard, Berkeley, California, 94708.

PUBLISHED BY PACIFIC JOURNAL OF MATHEMATICS, A NON-PROFIT CORPORATION

Printed at Kokusai Bunken Insatsusha (International Academic Printing Co., Ltd.), 8-8, 3-chome, Takadanobaba, Shinjuku-ku, Tokyo 160, Japan. 


\section{Pacific Journal of Mathematics \\ Vol. 65, No. $2 \quad$ October, 1976}

Andrew Adler, Weak homomorphisms and invariants: an example .......... 293

Howard Anton and William J. Pervin, Separation axioms and metric-like

functions ............................................. 299

Ron C. Blei, Sidon partitions and p-Sidon sets .................... 307

T. J. Cheatham and J. R. Smith, Regular and semisimple modules ........... 315

Charles Edward Cleaver, Packing spheres in Orlicz spaces .............. 325

Le Baron O. Ferguson and Michael D. Rusk, Korovkin sets for an operator on a

space of continuous functions ............................. 337

Rudolf Fritsch, An approximation theorem for maps into Kan fibrations ....... 347

David Sexton Gilliam, Geometry and the Radon-Nikodym theorem in strict

Mackey convergence spaces .................................

William Hery, Maximal ideals in algebras of topological algebra valued

functions ...................................... 365

Alan Hopenwasser, The radical of a reflexive operator algebra ........... 375

Bruno Kramm, A characterization of Riemann algebras................. 393

Peter K. F. Kuhfittig, Fixed points of locally contractive and nonexpansive

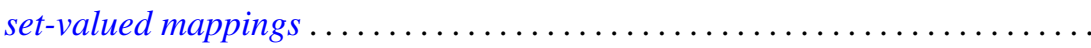

Stephen Allan McGrath, On almost everywhere convergence of Abel means of

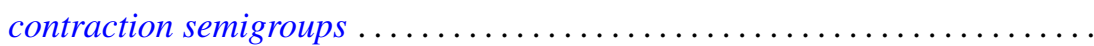

Edward Peter Merkes and Marion Wetzel, A geometric characterization of

indeterminate moment sequences............................ 409

John C. Morgan, II, The absolute Baire property ................... 421

Eli Aaron Passow and John A. Roulier, Negative theorems on generalized convex approximation .................................... 437

Louis Jackson Ratliff, Jr., A theorem on prime divisors of zero and characterizations of unmixed local domains ..............

Ellen Elizabeth Reed, A class of $T_{1}$-compactifications................... 471

Maxwell Alexander Rosenlicht, On Liouville's theory of elementary

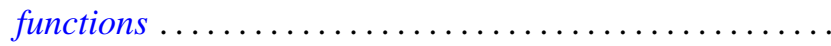

Arthur Argyle Sagle, Power-associative algebras and Riemannian

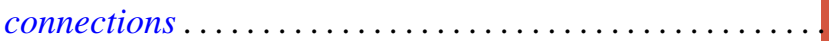

Chester Cornelius Seabury, On extending regular holomorphic maps from Stein manifolds...

Elias Sai Wan Shiu, Commutators and numerical ranges of powers of operators ...................................

Donald Mark Topkis, The structure of sublattices of the product of $n$ lattices ... 525

John Bason Wagoner, Delooping the continuous $K$-theory of a valuation

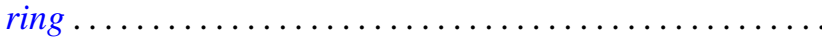

Ronson Joseph Warne, Standard regular semigroups...........

Anthony William Wickstead, The centraliser of $E \otimes_{\lambda} F \ldots$. 